\title{
Design and Evaluation of a Sensitive, Low Cost and Portable Millimeter Wave Survey Meter
}

\section{Howard Bassen', Negin Shahshahan'2, Amir Razjouyan', Gonzalo Mendoza ${ }^{3}$, Mohammed Eslami ${ }^{4}$, Trent Robertson ${ }^{3}$}

${ }^{1}$ US Food and Drug Administration, Center for Devices and Radiological Health,

Silver Spring, MD, USA

${ }^{2}$ Drexel University College of Medicine, Philadelphia, PA, USA

${ }^{3}$ Chickasaw Nation Industries, Norman, OK, USA

${ }^{4}$ Netrias, LLC, Bethesda, MD, USA

Email: howard.bassen@fda.hhs.gov

How to cite this paper: Bassen, H., Shahshahan, N., Razjouyan, A., Mendoza, G., Eslami, M. and Robertson, T. (2018) Design and Evaluation of a Sensitive, Low Cost and Portable Millimeter Wave Survey Meter. Open Journal of Antennas and Propagation, 6, 25-35.

https://doi.org/10.4236/ojapr.2018.63003

Received: August 2, 2018

Accepted: September 15, 2018

Published: September 18, 2018

Copyright (C) 2018 by authors and Scientific Research Publishing Inc. This work is licensed under the Creative Commons Attribution International License (CC BY 4.0).

http://creativecommons.org/licenses/by/4.0/

\begin{abstract}
We developed a small, hand-held, portable, low cost, millimeter wave ( $\mathrm{mmW}$ ) meter to detect and display the levels of emissions from L3 ProVision security scanners used in hundreds of airports for detection of contraband and weapons. The meter is intended to measure radiation emissions from this particular scanner and enable the user to enable periodic quality assurance measurements and to see if undesirably high levels were to occur. The non-engineering staff of the Transportation Security Administration (TSA) can use this meter to demonstrate safety of these scanners to passengers using this simple handheld instrument certified by the US Food Drug administration. This meter can assess human exposure levels during periodic quality assurance inspections, in a region where a person is being scanned, and demonstrate the lack of possible interference with body worn medical devices. It has receiving, signal processing, output display, and user control subsystems, and displays graphical and numerical information. The meter detects low level, pulses of $\mathrm{mmW}$ electromagnetic radiation $(20-30 \mathrm{GHz}$ with levels of $0.02 \mathrm{~V} / \mathrm{m}$ to $0.15 \mathrm{~V} / \mathrm{m}$ ). It displays a single burst of five or more $10 \mu$ sec pulses. It detects levels as low as several thousand times below the maximum permissible levels prescribed by international human exposure safety standards. This compact system replaces the bulky and costly collection of large and costly instruments needed to perform the same measurements.
\end{abstract}

\section{Keywords}

Index Terms Millimeter Wave, Measurement, Instrument, Meter, Exposure 


\section{Introduction}

The ProVision by L-3 Communications Inc. [1] is a millimeter wave (mmW) body scanner system that is widely used in airports to scan passengers for contraband. The second-generation system is termed the advanced imaging technology (AIT-2) system. These security systems emit mmW electromagnetic radiation in the range of $20-30 \mathrm{GHz}$. There have been certain public concerns expressed about exposures to millimeter wave emissions from these scanners and potential electromagnetic interference (EMI) of body worn and implanted medical devices or about human exposures [2] [3]. The transportation security administration wanted to address these concerns from airline passengers and from employees who could be exposed during installation, testing and use of the scanners, where repeated exposures or extended exposure durations occur. This led to an interagency agreement between the Transportation Security Administration (TSA) and the U.S. Food and Drug Administration (FDA), Center for Devices and Radiological Health (CDRH) to assess the safety of AIT systems. In 2011-2012 FDA performed a detailed study of the first-generation Advanced Imaging Technology system (AIT-1) that included measurements of emissions from an actual AIT system and tests of several different implanted or body worn medical devices. In 2015 and 2016 FDA performed a study of the second-generation scanner (AIT-2). It should be noted that operating parameters of the AIT system listed in this paper are not exact because this information is considered sensitive by the TSA. The FDA performed laboratory measurements of $\mathrm{mmW}$ emissions with a collection of large and costly meters comprising a detection system consisting of a receiving antenna, a low-noise $\mathrm{mmW}$ amplifier, a $\mathrm{mmW}$ diode detector and a low noise post-detection amplifier. This procedure was done because there are no portable instruments presently available with the needed capabilities for spatial, time, and field strength measurements. The findings from the initial AIT-1 study are contained in a report prepared by engineers in the Electromagnetics and Wireless Laboratory of the FDA and are published within a larger report from the TSA [4]. The findings to assess potential effects on active medical devices (devices powered by batteries) revealed that none of the medical devices under test experienced malfunctions or other effects from exposure to the AIT-1 security system. In addition, the report analyzed human exposures and concluded that $\mathrm{mmW}$ scanners currently deployed in the U.S. expose passengers and operational personnel to radiation levels that are much less than 1000 times below the applicable safety standards [5] [6]. Nonetheless, the safety of $\mathrm{mmW}$ scanners is still questioned by some.

The goal of our work was to develop a portable, stand-alone, low-cost measurement instrument that can detect the weak, transient emissions from L3 millimeter wave body scanners. Therefore, a quality assurance measurement system was developed to assess human exposure levels during periodic quality assurance inspections, in a region where a person is being scanned, and to identify the lack of possible interference with body worn medical devices. The non-engineering 
staff of the Transportation Security Administration (TSA) can use this meter to demonstrate safety of these scanners to passengers with minimal training of the TSA staff. The meter is a simple handheld instrument with calibration certified by the US Food Drug administration. Our work fills the void in available self-contained, portable measurement devices for detecting short pulse millimeter wave fields of very low amplitude. The key differences between our work and existing related devices are as follows. There are numerous self-contained, low-cost portable instruments for measuring electromagnetic fields in the lower $\mathrm{RF}$ and the microwave frequency bands but these cannot cover our frequency range of interest [7] [8] [9] [10] [11]. Alternatively, there are a very few commercially available portable radiation survey instruments with $\mathrm{mmW}$ detection capabilities. They have a very high cost and do not have the sensitivity or response time necessary to detect a short burst of $10 \mu \mathrm{sec}$ single pulses from millimeter wave body scanners [12]. The novelty and advances that our work possesses in terms of instrumentation and measurement include addressing a new and unique challenge. This challenge involves measuring and displaying the instantaneous peak field strengths emitted by millimeter wave airport body scanners in a low cost, self-contained and portable device. It is not a simple task to transition the lower frequency instruments with their long response times to a very fast detector of millimeter waves using low cost components. The device does not use any new technologies but rather implements existing technologies in a much lower cost manner than used in any previous devices.

The purpose of this paper is to describe the successful development of a low cost, portable, hand-held millimeter wave $(\mathrm{mmW})$ survey meter. This meter can measure the levels of $\mathrm{mmW}$ radiation at various locations in and around L3 AIT security scanners and indicate if the emissions exceed expected, normal operating levels (field strengths greater than $0.15 \mathrm{~V} / \mathrm{m}$ ). Our device operates without the need for an assortment of large and costly electronic instruments. The meter does not require any expertise to use for interpretation of exposure levels. AIT security systems have radiating antennas that sweep around the person being scanned, exposing them to many very low-level pulses of mmW energy over a period of about 1.6 seconds. The meter can be used to detect the presence of weak mmW emissions from the AIT-1 and AIT-2 security scanners inside, at the entrance, or at the exit of the scanner. It can indicate that emissions are at the expected, normal level (over 1000 times less than the maximum permissible levels of prevailing electromagnetic radiation safety standards). The meter displays graphical plots of the individual $\mathrm{mmW}$ bursts from AIT security system and samples and holds a numerical value for the maximum value of the field strength. The display is based on calibrations from FDA's precise laboratory standards of $\mathrm{mmW}$ field strengths. The intended use would be to provide one or more of these meters at every airport where a scanner is used for periodic quality assurance tests and to demonstrate safety to passengers on demand. 
This paper will discuss:

1) Methods and Materials;

2) Testing and Calibration of the $\mathrm{mmW}$ meter;

3) Conclusions and Future Work.

\section{Methods and Materials}

The overall meter is composed of subsystems as shown in Figure 1 and is described below. Our overall goal for the meter was to produce a very low-cost system, which required an innovative approach to developing low-cost components and signal processing techniques. It should be noted that certain information about the AIT and data are generalized since the security of the L3 security scanning system requires protection from those that might try to bypass its capabilities.

1) Millimeter Wave Meter Receiving Subsystem-The $\mathrm{mmW}$ meter Receiving subsystem is shown in Figure 2. It is designed to capture the fields from the AIT radiating elements in the antenna arms as they sweep around a person being scanned in approximately 1.6 seconds. The AIT emissions produce a burst of $\mathrm{mmW}$ pulses that is received by the survey meter antenna portion of this subsystem. The subsystem captures the weak millimeter wave signal via a 3-D printed metal receiving waveguide horn antenna with a gain of $20 \mathrm{~dB}$. This antenna was designed in the FDA's Additive Manufacturing of Medical Products

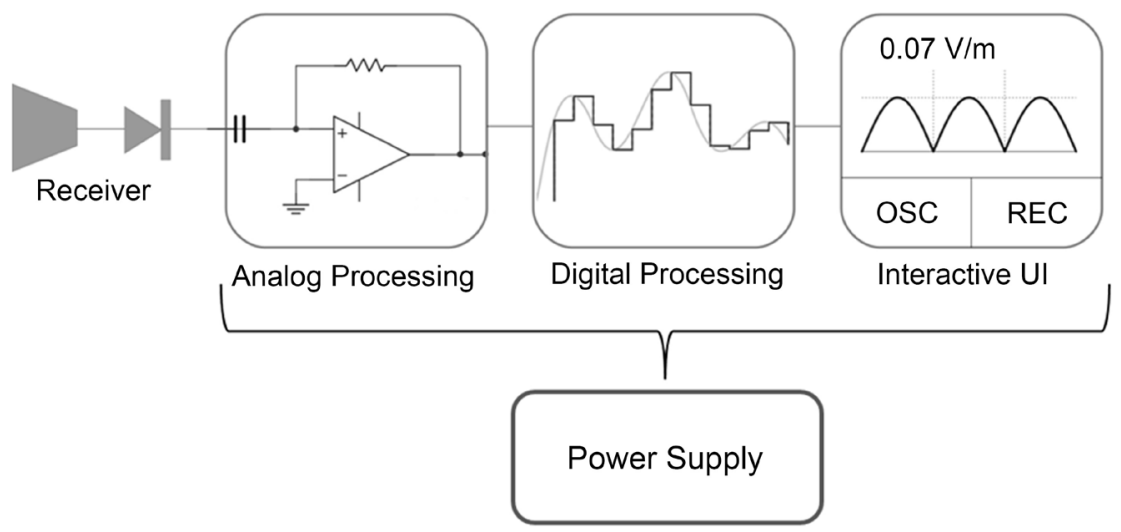

Figure 1. Block diagram of the mmW meter.

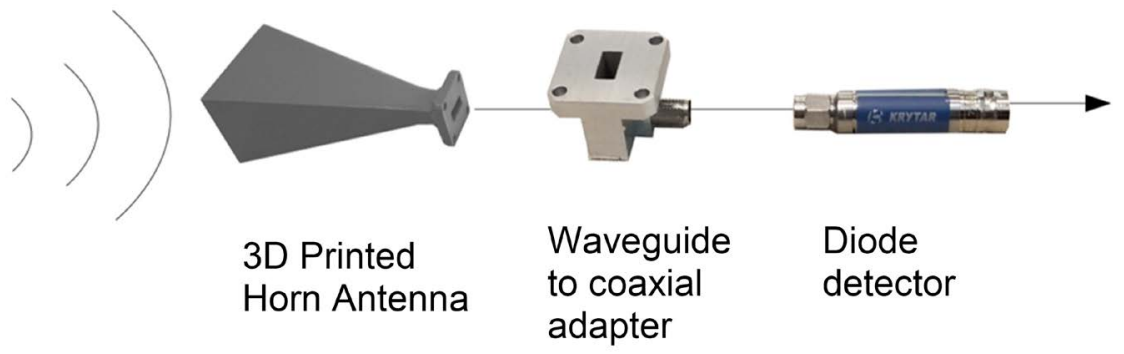

Figure 2. mmW receiving subsystem. 
(AMMP) Lab, and produced by GPI Prototype \& Manufacturing Services Inc. Lake Bluff, IL 60,044. It costs much less than commercially available horn antennas in quantities of 10 . The antenna is designed for the frequency range of about $20-30 \mathrm{GHz}$. This antenna was designed in accordance with standard gain horn antenna specifications and its performance is very similar to the commercially available millimeter wave horn antennas we used in our previous studies of the L3 AIT-1.

The antenna is connected to a mmW diode detector (see component specification in Table 1) via a waveguide to coaxial adapter. The diode detector converts the weak pulses of $\mathrm{mmW}$ power from the antenna to low frequency voltage pulses. The output of the mmW receiving subsystem serves as the input to the low-frequency analog electronic signal processing subsystem. The AIT emissions are amplitude modulated with a complex pulsed waveform that has many brief duration pulses contained within a larger pulse burst. The "ON" time of the longer bursts is several milliseconds. The output amplitude from the diode detector is in the microvolt range. The output of the $\mathrm{mmW}$ receiving subsystem is then transmitted to the input of the analog electronic signal processing subsystem.

2) Electronic Signal Processing Subsystem-The signal processing subsystem consists of an analog subsystem and a digital subsystem. The analog electronic signal processing subsystem receives a weak voltage that is proportional to the instantaneous peak pulsed electric (E) field strength seen by the $\mathrm{mmW}$ meter's antenna. The analog subsystem consists of a circuit that performs amplification, filtering and envelope detection of pulsed voltages from the diode detector. The analog electronic signal processing subsystem (Figure 3 ) is configured with an input stage (preamplifier) consisting of two operational amplifiers (AD827, Analog devices Inc.) that amplify the weak input signal in a low-pass frequency band of several hundred $\mathrm{kHz}$. This stage is followed by an active analog filter circuit composed of two AD827 operational amplifiers that have a bandpass of $20 \mathrm{kHz}$ centered at the frequency of interest. This filter reduces the noise accompanying the received signal significantly. The output of the filter is followed by a true RMS chip (AD636JD, Analog Devices Inc.) that acts as an envelope detector and low-pass filter. It produces a smooth unipolar envelope from the many short pulses delivered to its input within the large overall pulsed emission burst of the AIT scanner. The frequency range of this output signal is well within the maximum sampling rate of the digital processing circuit that

Table 1. Millimeter wave components.

\begin{tabular}{cc}
\hline Component & Brand/Model \# \\
\hline Horn Antenna & Custom 3D printed metal antenna. GPI Prototype \& \\
& Manufacturing Services Inc. \\
Waveguide to coaxial adapter & Pasternack PEWCA1021 \\
Schottky Diode Detector & Krytar 203 BK \\
\hline
\end{tabular}




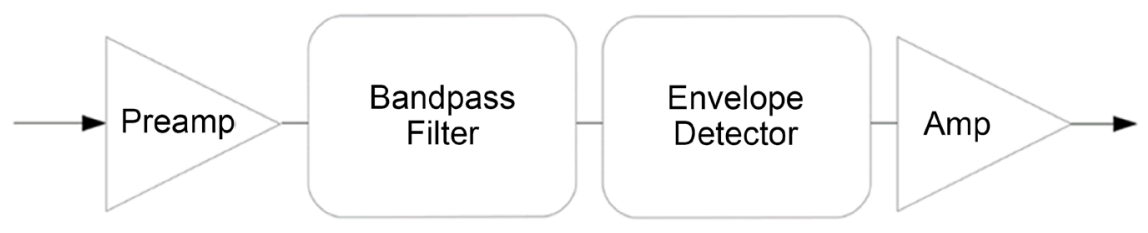

Figure 3. Analog electronic signal processing subsystem.

follows. This is amplified by a low frequency post detection amplifier with low gain. It uses an operational amplifier (AD827) to raise the processed signal into the input amplitude range of the analog-to-digital converter of the digital processing subsystem that follows. The analog input circuit design was implemented on a four-layer printed circuit board with a solid copper ground plane to produce low noise performance. During initial development of the meter, problems were encountered with noise and oscillations on the circuit signals that were traced to issues with the layout of ground conductors and longer wires. These issues were solved by using a printed circuit board with adequate decoupling capacitors on the voltage supply pins of each circuit chip, and using careful grounding techniques [13].

The digital signal processing subsystem is fed with the output signal of the analog system. It consists of a low-cost microcontroller development board model (PJRC.com) with a 32-bit ARM Cortex-M4 microprocessor and a 180 $\mathrm{MHz}$ clock. It is programmed using the $\mathrm{C}$ computer language. Microprocessor operations include analog data acquisition and digitization, filtering, peak detection, data logging and data storage plus output to a graphic display. Data are displayed graphically in real time in terms of signal strength received versus time and is stored on an SD memory card that can be played back on the meter itself or displayed on a personal computer. A flow diagram of the software for digital signal processing is shown in Figure 4.

\section{3) Output Display and User Control Subsystem}

This stage consists of a 3.5" color liquid crystal display (LCD) with touch interactivity. It receives digital input data from the digital signal processing subsystem and displays a graphical waveform of the signal being received in a storage oscilloscope type display. Text data is also displayed including the numerical value of the peak detected voltage (Figure 5). Additional display modes can display the actual volts per meter. User controls include touch screen features on the LCD display for selecting different functions including the starting and stopping of data acquisition and the recall and playback of graphical data displayed.

\section{4) Mechanical Construction and Power Supply}

The system is completely portable and self-contained and can be brought to the scanner location that needs to be evaluated. The system is housed in an aluminum box with dimensions of $15 \times 10 \times 5 \mathrm{~cm}$, with the $\mathrm{mmW}$ horn antenna mounted on the outside of the box. The mmW diode detector diode and printed circuit boards are mounted inside the box along with the batteries. Two 


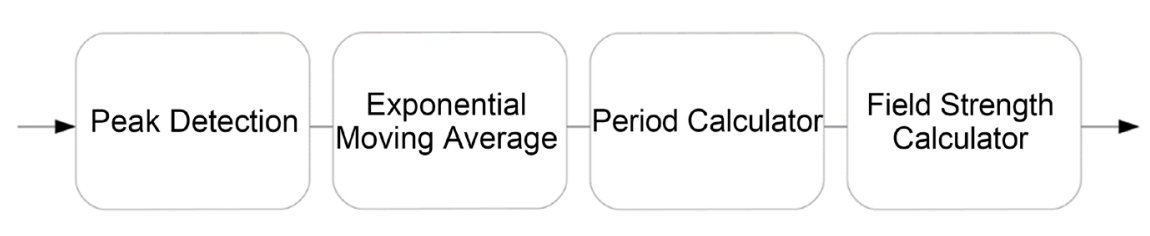

Figure 4. Digital signal processing flow diagram.

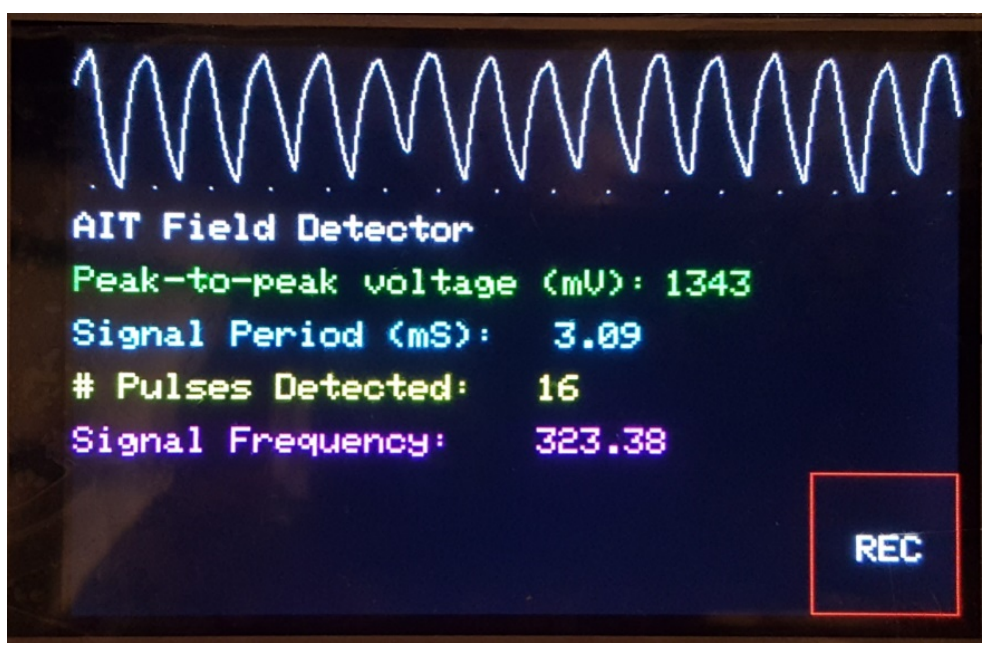

Figure 5. mmW meter output display and user control.

rechargeable 9-volt $\mathrm{NiMH}$ batteries and a 3.7-volt lithium ion polymer battery are incorporated in the box. Figure 6 shows the prototype meter's construction.

\section{Testing and Calibration of the mmW Meter}

The survey meter was designed, calibrated and tested primarily using a novel AIT simulation system developed by FDA to produce emissions very similar to the actual AIT scanner [4]. The simulation system was used because access to AIT systems was very limited and development and refinement of the survey meter using AIT emissions took many hours. However, several tests and measurements were performed with the actual AIT systems to verify real world performance. The AIT simulation system can produce exposure fields that simulate the strength and pulse characteristics of an actual mmW AIT scanner. The AIT simulator consists of the components shown in Figure 7 and Table 2. The simulator system uses a voltage controlled $\mathrm{mmW}$ oscillator (sweeping from approximately $20-30 \mathrm{GHz}$ ) along with an arbitrary wave generator to produce a $\mathrm{mmW}$ carrier signal with square wave modulation of about $100 \mathrm{kHz}$. The $\mathrm{mmW}$ signal is then fed to a transmitting horn antenna to replicate the emissions from the L3 security system's antennas. During its evaluation, the face of the horn antenna of the mmW meter was placed $70 \mathrm{~cm}$ away from the AIT simulator's radiating antenna along the boresight of the simulator's antenna. An exposure level for the simulator was set at $0.02 \mathrm{~V} / \mathrm{m}$ at the location of the meter's receiving antenna and a burst of five $10 \mu \mathrm{sec}$ pulses was generated by the simulator. The meter displayed the burst momentarily on the graphical display as a line plot and 


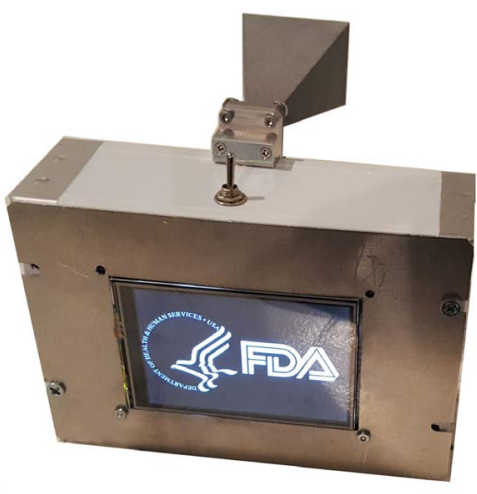

(a)

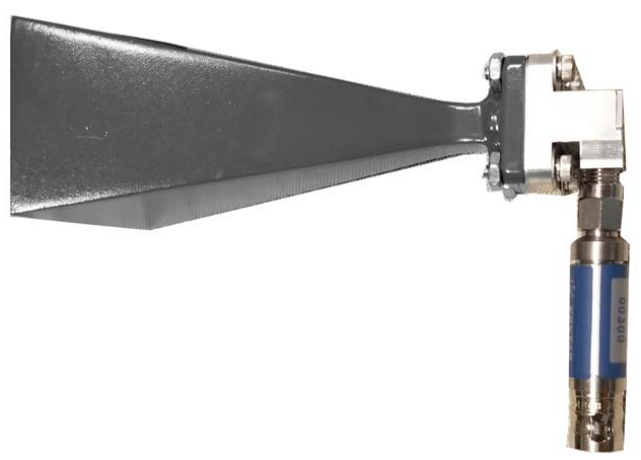

(b)

Figure 6. The mmW meter hardware. (a) Overall system; (b) Front end: antenna and diode detector.

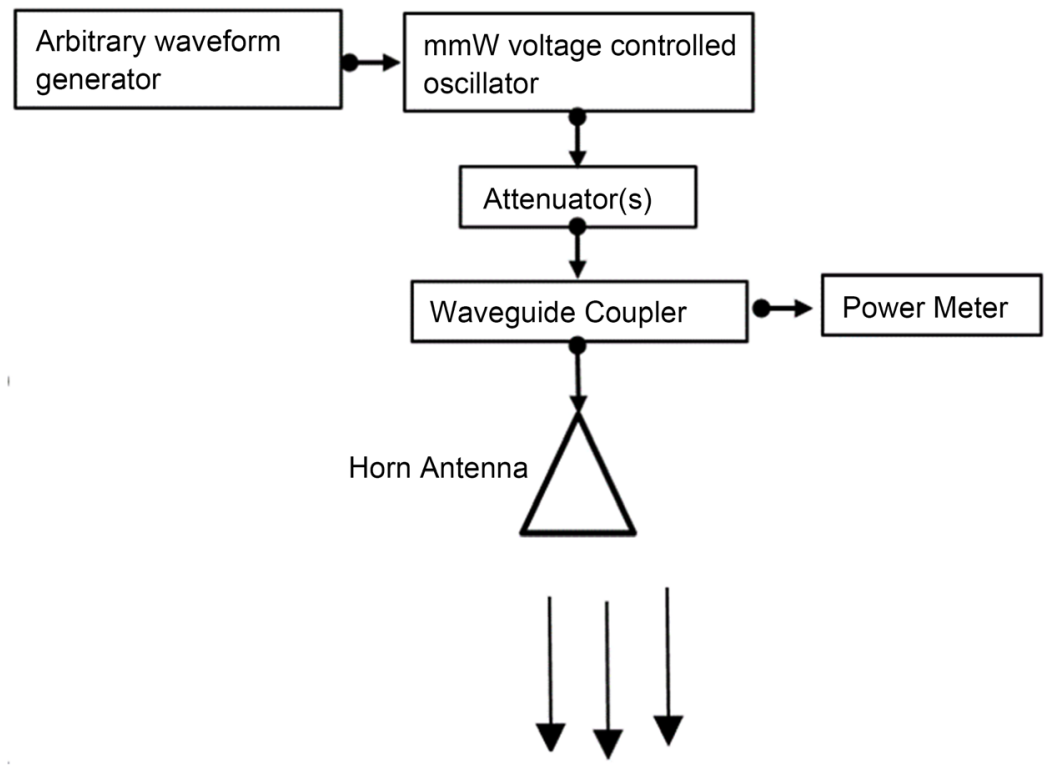

Figure 7. AIT simulator (arrows represent mmW output fields).

Table 2. Components in the mmW simulator used to test the meter.

\begin{tabular}{cc}
\hline Component & Brand/Model \# \\
\hline Arbitrary waveform generator & Agilent E 33522A \\
mmW Signal Generator & Agilent MGX-N5183A \\
Attenuator & Mini-Circuits \\
Baveguide Coupler & BW-S20W5/VAT-10W2+ \\
Power Meter & Agilent E4419B \\
Horn Antenna & Pasternack PE9851-20 \\
\hline
\end{tabular}

digital values. This test was used to calibrate the meter. After calibration and during its use, the meter's peak value in volts per meter was captured and dis- 
played as a numerical value on the survey meter's LCD screen. In addition, the data were stored in the meter's memory for immediate playback of the waveform and for later analysis.

In another test, a comparison was made between the 3D printed antenna and a commercially available $20 \mathrm{~dB}$ gain horn antenna of the same physical dimensions. The same sensitivity was achieved with the 3-D printed antenna within 0.5 $\mathrm{dB}$. The pattern of both horn antennas was basically identical since the 3-D printed antenna was designed with the exact dimensions of the commercially available antenna. The relatively narrow beam with of the meter's antenna is not a problem for detection of millimeter wave fields emitted the L3 body scanner. This is because the scanner sweeps the $180^{\circ}$ arc around the location of the person being scanned, thus passing the beam of the millimeter waves through the boresight of the receiving antenna.

The performance of the handheld, portable mmW meter was also tested with an actual AIT-2, the L3 ProVision 2 Compact Advanced Personnel Screening system at a TSA test lab near the Ronald Reagan Washington National Airport outside Washington, D.C. The meter correctly sensed a burst of pulsed fields from the scanner as it swept one time around the spot where a passenger stands when being scanned. During this testing of the $\mathrm{mmW}$ meter checks were performed at other locations around the AIT-2 security system. The data were recorded in system memory and were analyzed later and used to assess the meter's performance. Several refinements were incorporated in the survey meter software based on the results of these AIT tests to improve performance.

\section{Conclusions and Future Work}

A novel, compact, hand-held, portable and low cost mmW survey meter was designed with the capacity to detect low intensity pulsed fields emitted by the L3 AIT scanning system. The data from the meter's measurement are intended to determine if AIT emissions exceed a set threshold over a range of $\mathrm{mmW}$ frequencies. Specifically, it can detect and display graphically, real-time signals between $0.02 \mathrm{~V} / \mathrm{m}$ and $0.15 \mathrm{~V} / \mathrm{m}$, with a pulse modulation of about $100 \mathrm{kHz}$ and with durations of $10 \mu \mathrm{sec}$ or more. The meter provides a low cost, portable, compact, hand-held means to detect and characterize weak, short duration pulses of mmW fields in the proximity of a mmW AIT security scanner for periodic quality assurance checks. The meter also can demonstrate to passengers who question the safety of scanner that extremely low levels exist as measured by an FDA certified instrument. The low cost of this device makes it feasible for deployment at many hundreds of airports for use by the non-engineering staff of the Transportation Security Administration (TSA) with minimal training. The project utilized a low-cost circuit design, a 3D printed metal antenna, and a low-cost microprocessor for digital signal processing to reduce the need for expensive components. The sensitivity of the prototype meter is $0.02 \mathrm{~V} / \mathrm{m}$ for AIT pulses, as verified with tests in our mmW AIT simulator system. The design of 
the $\mathrm{mmW}$ meter required measuring a weak signal from a $\mathrm{mmW}$ diode detector with a broadband noise level of about $0.1 \mathrm{mV}$. The design of the detection circuit incorporates amplification of this signal with two stages of low frequency gain with a narrow bandwidth around $20 \mathrm{kHz}$. Additional narrowing of the bandwidth in the post detection stages of the analog subsystem, and signal processing in the digital subsystem make detection of $0.02 \mathrm{~V} / \mathrm{m}$ possible.

Future work includes evaluating the feasibility for redesign and refinement to enable using the meter with other body imaging security scanners that operate with emissions ranging from 10 to $100 \mathrm{GHz}$. Such refinements would require using different receiving antennas and detector diodes so that the meter could cover a wide range of frequencies and field strengths. In addition, changes to the signal processing circuitry and bandpass filters would have to be developed for each specific scanner. This would allow detection of weak, pulsed microwave and millimeter wave fields from other scanners.

\section{Acknowledgements}

This work was partially supported by the Transportation Security Administration through Interagency Agreement HSTS04-12-X-CT2000 (FDA IAG 224-12-6014). This project was supported in part by an appointment to the Research Participation Program at the Office of Science and Engineering Laboratories, Center for Devices and Radiological Health, U.S. Food and Drug Administration, administered by the Oak Ridge Institute for Science and Education through an interagency agreement between the U.S. Department of Energy and FDA.

The mention of commercial products, their sources, or their use in connection with material reported herein is not to be construed as either an actual or implied endorsement of such products by the Department of Health and Human Services.

\section{Conflicts of Interest}

The authors declare no conflicts of interest regarding the publication of this paper.

\section{References}

[1] L3 Security \& Detection Systems. http://www.sds.1-3com.com/advancedimaging/provision-2.htm

[2] International Commission on Non-Ionizing Radiation Protection, ICNIRP Guidelines (2012) ICNIRP Statement-Health Issues Associated with Millimeter Wave Whole Body Imaging Technology. Health Physics, 102, 81-82.

[3] Accardo, J. and Chaudhry, M.A. (2014) Radiation Exposure and Privacy Concerns Surrounding Full-Body Scanners in Airports. Journal of Radiation Research and Applied Sciences, 7, 198-200. https://doi.org/10.1016/j.jrras.2014.02.005

[4] U.S. Department of Homeland Security, Science and Technology Directorat (2012) Compilation of Emission Safety Reports on the L3 Communications, Inc. ProVision 100 Active Millimeter Wave, Advanced Imaging Technology (AIT) System, Version 
2. DHS/ST/TSL-12/118.

https://www.dhs.gov/sites/default/files/publications/tsa-compilation-of-emission-sa fety-reports-on-the-13-communications-inc-ait-system.pdf

[5] Institute of Electrical and Electronics Engineers (2006) IEEE Standard C95.1-2006 for Safety Levels with Respect to Human Exposure to Radio Frequency Electromagnetic Fields, $3 \mathrm{kHz}$ to $300 \mathrm{GHz}$.

[6] International Commission on Non-Ionizing Radiation Protection (ICNIRP) (1998) Guidelines for Limiting Exposure to Time-Varying Electric, Magnetic, and Electromagnetic Fields (up to $300 \mathrm{GHz}$ ). Health Physics, 74, 494-522.

[7] Aslan, Edward, A. (1972) Broad-Band Isotropic Electromagnetic Radiation Monitor. IEEE Transactions on Instrumentation and Measurement, 21, 421-424. https://doi.org/10.1109/TIM.1972.4314059

[8] Aslan, Edward, A. (1970) Electromagnetic Radiation Survey Meter. IEEE Transactions on Instrumentation and Measurement, 19, 368-372. https://doi.org/10.1109/TIM.1970.4313930

[9] Kanda, M. (1993) Standard Probes for Electromagnetic Field Measurements. IEEE Transactions on Antennas and Propagation, 41, 1349-1364. https://doi.org/10.1109/8.247775

[10] Munter, K., Pape, R., Glimm, J. and Portable, E. (1997) Field Strength Meter and Its Traceable Calibration up to $1 \mathrm{GHz}$ Using a uTEM Cell. IEEE Transactions on Instrumentation and Measurement, 46, 549-550. https://doi.org/10.1109/19.571908

[11] Yarovoy, A., de Jongh, R. and Ligthart, L. (2000) Ultra-Wideband Sensor for Electromagnetic Field Measurements in Time Domain. Electronics Letters, 36, 1679-1680. https://doi.org/10.1049/el:20001171

[12] NBM E-Field-Probe EF 5091, Narda Safety Test Solutions. Hauppauge, NY, USA.

[13] Zumbahlen, H. (2012) Staying Well Grounded. Vol. 46, Analog Dialogue. http://www.analog.com/library/analogDialogue/archives/46-06/staying_well_groun ded.html 